\title{
Parkinson's Disease: Progress and Controversies
}

The continuing search for causes and effective treatments or prevention of chronic neurodegenerative diseases is slow and frustrating, particularly for the families and patients affected by these disorders. Some encouragement is provided by Parkinson's disease (PD) which, in some respects, can be considered as a model neurodegenerative disease and a partial success story at this point in time.

It is less than 25 years since striatal dopamine deficiency was identified as the major neurochemical abnormality responsible for the motor deficits of $\mathrm{PD}$. This observation was soon followed by the first clinical trials using oral levodopa as replacement therapy. Progress since then has been quite remarkable, particularly during the past decade. New levodopa preparations and direct dopamine agonists have undergone clinical trials and become available for routine use. The MPTP story has generated a great deal of excitement and has led to a renewed search for environmental factors which may contribute to the pathogenesis of idiopathic PD.

Studies using animal models have clearly indicated that transplantation of neural tissue can restore functions which have been lost as a result of damage to selected neuronal populations. It is now apparent that the initial enthusiasm for auto-transplantation with adrenal medullary tissue in patients with PD was premature and not based on solid scientific principles. However, carefully designed studies transplanting human embryonic tissue are now in progress and preliminary results are encouraging. With the enthusiasm surrounding these developments, it is easy for clinicians to become complacent and ignore two obvious problems - we still do not know what factors are responsible for degeneration and death of neurons in idiopathic PD, and the disease continues to progress despite symptomatic treatment with levodopa preparations and dopamine agonists.
The Parkinson's disease story has been well documented in the Canadian Journal of Neurological Sciences. In addition to numerous original articles dealing with a variety of different aspects of the disease, the Journal has published two special supplements during the past 6 years. The first, in 1984, entitled "Current Concepts and Controversies in Parkinson's Disease" included one of the initial reports on MPTP parkinsonism by Langston. Three years later in 1987 a supplement entitled "Disorders of Muscle Tone" highlighted the possible role of environmental toxins.

In this issue of the Journal we present several features which address some of the problems outlined above. The possible roles of environmental factors and genetics are reviewed by Poirier et al. In another review article Furtado and Mazurek discuss mechanisms of MPTP neurotoxicity and the rationale for preventive therapy with agents such as deprenyl. Initial reports on the possible beneficial effects of deprenyl have generated numerous questions and controversies, and two opposing views are provided in commentaries by Oscar Kofman and David Grimes. Finally, we include a summary of the discussions from a National Conference on Parkinson's Disease which was held in Victoria, British Columbia in September 1990.

The Parkinson's story is still far from complete. There are many problems and unanswered questions. But as we enter the second year of the Decade of the Brain, there are solid grounds for optimism that PD will be one of the chronic neurodegenerative disorders for which effective solutions will be found by the year 2000 .

Robert G. Lee, M.D. Editor 